TRANSACTIONS OF THE

AMERICAN MATHEMATICAL SOCIETY

Volume 350, Number 12, December 1998, Pages 4839-4847

S 0002-9947(98)02359-9

\title{
BAIRE AND $\sigma$-BOREL CHARACTERIZATIONS OF WEAKLY COMPACT SETS IN $M(T)$
}

\author{
T. V. PANCHAPAGESAN
}

\begin{abstract}
Let $T$ be a locally compact Hausdorff space and let $M(T)$ be the Banach space of all bounded complex Radon measures on $T$. Let $\mathcal{B}_{o}(T)$ and $\mathcal{B}_{c}(T)$ be the $\sigma$-rings generated by the compact $G_{\delta}$ subsets and by the compact subsets of $T$, respectively. The members of $\mathcal{B}_{o}(T)$ are called Baire sets of $T$ and those of $\mathcal{B}_{c}(T)$ are called $\sigma$-Borel sets of $T$ (since they are precisely the $\sigma$-bounded Borel sets of $T$ ). Identifying $M(T)$ with the Banach space of all Borel regular complex measures on $T$, in this note we characterize weakly compact subsets $A$ of $M(T)$ in terms of the Baire and $\sigma$-Borel restrictions of the members of $A$. These characterizations permit us to give a generalization of a theorem of Dieudonné which is stronger and more natural than that given by Grothendieck.
\end{abstract}

\section{INTRODUCTION}

For a locally compact Hausdorff space $T$, let $C_{o}(T)$ be the Banach space of all continuous complex functions vanishing at infinity in $T$, endowed with the supremum norm. Let $\mathcal{B}(T)$ be the $\sigma$-algebra of Borel sets in $T$. The dual $M(T)$ of $C_{o}(T)$ is the Banach space of all bounded complex Radon measures $\mu$ on $T$ and by Theorem 5.3 of [P2] is isometrically isomorphic to the Banach space of all Borel regular complex measures $\mu$ on $\mathcal{B}(T)$, endowed with the norm $\|\mu\|=|\mu|(T)$, where $|\mu|$ denotes the variation of $\mu$ in $\mathcal{B}(T)$. Hence, we identify $M(T)$ with the Banach space of all Borel regular complex measures on $\mathcal{B}(T)$. Let $X$ be a quasicomplete locally convex Hausdorff space (briefly, a quasicomplete lcHs).

In the present note we characterize weakly compact sets $A$ in $M(T)$ in terms of the Baire and $\sigma$-Borel restrictions of the members of $A$. As a consequence of the Baire characterizations, we obtain a generalization of Proposition 8 of Dieudonné [Die], which is stronger and more natural than that of Grothendieck given on p. 150 of $[\mathrm{G}]$.

These characterizations will be powerful enough to replace the use of Theorem 3 and Proposition 11 of [G] in the study of weakly compact operators [P3]. In fact, these results play a key role in [P3] to provide a unified approach to the study of

Received by the editors November 17, 1995.

1991 Mathematics Subject Classification. Primary 28A33, 28C05, 28C15; Secondary 46E27.

Key words and phrases. Bounded complex Radon measures, uniform $\sigma$-additivity, uniform Baire inner regularity, uniform $\sigma$-Borel inner regularity, uniform Borel inner regularity, weakly compact sets.

Supported by the C.D.C.H.T. project C-586 of the Universidad de los Andes, Mérida, and by the international cooperation project between CONICIT-Venezuela and CNR-Italy.

(C)1998 American Mathematical Society 
weakly compact operators $u: C_{o}(T) \rightarrow X$ and of regular Borel extension of $X$ valued $\sigma$-additive Baire measures on $T$. In this context we would like to point out that the study of weakly compact operators was carried out by Grothendieck $[\mathrm{G}]$ for complete lcHs-valued operators on $C_{o}(T)$, and by Bartle, Dunford and Schwartz [BDS] for Banach space valued operators on $C(\Omega), \Omega$ compact, while the regular Borel extension problem for quasicomplete lcHs-valued Baire measures was studied by Dinculeanu and Kluvánek in [DK, K] by vector measure methods. As far as we know, such a unification study has not been presented earlier in the literature.

\section{Preliminaries}

In this section we fix notation and terminology and also give some definitions and results which will be needed in the sequel.

Let $T$ be a locally compact Hausdorff space and let $C_{o}(T)$ be the Banach space of all complex continuous functions vanishing at infinity in $T$, endowed with the supremum norm. Let $\mathcal{B}(T)$ be the $\sigma$-algebra of Borel sets in $T$, which is the $\sigma$ algebra generated by the class of all open sets in $T$. Then the dual of $C_{o}(T)$ is the Banach space $M(T)$ of all bounded complex Radon measures $\mu$ on $T$, and by Theorem 5.3 of [P2] it is isometrically isomorphic to the Banach space of all Borel regular complex measures $\mu$ on $\mathcal{B}(T)$ endowed with the norm $\|\mu\|=|\mu|(T)$, where $|\mu|$ denotes the variation of $\mu$ in $\mathcal{B}(T)$. Consequently, we identify $M(T)$ with the Banach space of all Borel regular complex measures on $\mathcal{B}(T) . \mathcal{C}(T)\left(\right.$ resp. $\mathcal{C}_{o}(T)$ ) is the class of all compact subsets (resp. compact $G_{\delta}$ subsets) of $T . \mathcal{B}_{c}(T)$ (resp. $\mathcal{B}_{o}(T)$ ) is the $\sigma$-ring generated by $\mathcal{C}(T)$ (resp. by $\left.\mathcal{C}_{o}(T)\right)$ ), and the members of $\mathcal{B}_{c}(T)$ (resp. of $\left.\mathcal{B}_{o}(T)\right)$ are called $\sigma$-Borel (resp. Baire) sets of $T$. Since the members of $\mathcal{B}_{c}(T)$ are precisely the $\sigma$-bounded Borel sets of $T$, we call them $\sigma$-Borel.

We need the following lemma before we give some definitions.

Lemma 1. For $\mu \in M(T)$, let $|\mu|(\cdot)=\operatorname{var}(\mu,(\cdot))$ in $\mathcal{B}(T)$. Then

$$
\left.|\mu|\right|_{\mathcal{B}_{o}(T)}(\cdot)=\operatorname{var}\left(\left.\mu\right|_{\mathcal{B}_{o}(T)},(\cdot)\right) \text { and }\left.|\mu|\right|_{\mathcal{B}_{c}(T)}(\cdot)=\operatorname{var}\left(\left.\mu\right|_{\mathcal{B}_{c}(T)},(\cdot)\right) \text {. }
$$

Proof. Let $\mathcal{D}\left(\mathcal{C}_{o}(T)\right)$ be the $\delta$-ring generated by $\mathcal{C}_{o}(T)$. Then by Lemma 3.2 of [P2]

$$
|\mu|_{\mathcal{D}\left(\mathcal{C}_{o}(T)\right)}(\cdot)=\operatorname{var}\left(\left.\mu\right|_{\mathcal{D}\left(\mathcal{C}_{o}(T)\right)},(\cdot)\right)
$$

Given $E \in \mathcal{B}_{o}(T)$, there exists a disjoint sequence $\left(E_{n}\right)_{n=1}^{\infty} \subset \mathcal{D}\left(\mathcal{C}_{o}(T)\right)$ such that $E=\bigcup_{n=1}^{\infty} E_{n}$. Then

$$
\begin{aligned}
|\mu|(E) & =\sum_{n=1}^{\infty}|\mu|\left(E_{n}\right)=\sum_{n=1}^{\infty} \operatorname{var}\left(\left.\mu\right|_{\mathcal{D}\left(\mathcal{C}_{o}(T)\right)}, E_{n}\right) \\
& =\sum_{n=1}^{\infty} \operatorname{var}\left(\left.\mu\right|_{\mathcal{B}_{o}(T)}, E_{n}\right)=\operatorname{var}\left(\left.\mu\right|_{\mathcal{B}_{o}(T)}, E\right) .
\end{aligned}
$$

Now, let $E \in \mathcal{B}_{c}(T)$. Since it is easy to check that each $F \subset E$ with $F \in \mathcal{B}(T)$ is $\sigma$-Borel, it follows that $\mid \mu \|_{\mathcal{B}_{c}(T)}(E)=\operatorname{var}\left(\left.\mu\right|_{\mathcal{B}_{c}(T)}, E\right)$.

Notation 1. For $\mu \in M(T)$, let $|\mu|(\cdot)=\operatorname{var}(\mu,(\cdot))$ on $\mathcal{B}(T)$.

In the light of the above lemma, the variations used in the following definition are unambiguously defined. The first part is an adaptation of Definition 3.2 of [P1]. 
Definition 1. Let $\mathcal{S}$ be a $\sigma$-ring of sets in $T$ such that $\mathcal{C}(T) \subset \mathcal{S}$ or $\mathcal{C}_{o}(T) \subset \mathcal{S}$. A complex measure $\mu$ on $\mathcal{S}$ is said to be $\mathcal{S}$-regular if, given $E \in \mathcal{S}$ and $\varepsilon>0$, there exist a compact set $K \in \mathcal{S}$ and an open set $U \in \mathcal{S}$ with $K \subset E \subset U$ such that $|\mu(B)|<\varepsilon$ for every $B \in \mathcal{S}$ with $B \subset U \backslash K$. When $\mathcal{S}=\mathcal{B}(T)$ (resp. $\mathcal{B}_{c}(T), \mathcal{B}_{o}(T)$ ), we use the terminology Borel (resp. $\sigma$-Borel, Baire) regularity in place of $\mathcal{S}$-regularity. Let $A$ be a subset of $M(T)$. We say that $A$ is uniformly Baire inner regular (resp. Baire regular) in a set $E \in \mathcal{B}_{o}(T)$ if, given $\varepsilon>0$, there exists a compact $K \in \mathcal{B}_{o}(T)$ with $K \subset E$ (resp. and an open Baire set $O$ in $T$ with $K \subset E \subset O$ ) such that $\sup _{\mu \in A}|\mu|(E \backslash K)<\varepsilon$ (resp. such that $\left.\sup _{\mu \in A}|\mu|(O \backslash K)<\varepsilon\right)$. If $A$ is uniformly Baire inner regular (resp. Baire regular) in each Baire set, then $A$ is said to be uniformly Baire inner regular (resp. Baire regular). Similarly, the uniform Borel (resp. $\sigma$-Borel) regularity and inner regularity of $A$ and those of $A$ in a Borel (resp. $\sigma$-Borel) set $E$ are defined.

By virtue of Theorem 51.D of $[\mathrm{H}]$, we note that a compact $K \in \mathcal{B}_{o}(T)$ is necessarily a $G_{\delta}$.

It is well known that every complex Baire measure $\mu_{o}$ is Baire regular and that it has a unique extension $\mu$ to $\mathcal{B}(T)$ (resp. $\mu_{c}$ to $\mathcal{B}_{c}(T)$ ) such that $\mu$ is a Borel (resp. $\mu_{c}$ is a $\sigma$-Borel) regular complex measure. Moreover, $\left.\mu\right|_{\mathcal{B}_{c}(T)}=\mu_{c}$. (See, for example, Theorem 2.4 of [P2].)

Definition 2. A family $\mathcal{F}$ of complex measures defined on a $\sigma$-ring $\Sigma$ of sets is said to be uniformly $\sigma$-additive, if for each decreasing sequence $\left(E_{n}\right)$ of members of $\Sigma$ with $E_{n} \searrow \emptyset, \lim _{n} \mu\left(E_{n}\right)=0$ uniformly in $\mu \in \mathcal{F}$.

Notation 2. Given a $\sigma$-ring $\Sigma$ of sets, $c a(\Sigma)$ denotes the Banach space of all complex measures $\mu$ on $\Sigma$ with $\|\mu\|=\sup _{E \in \Sigma} \operatorname{var}(\mu, E)$.

The following result is well known when $\Sigma$ is a $\sigma$-algebra(see, for example, Theorem IV.9.1 of [DS]).

Proposition 1. Let $\Sigma$ be a $\sigma$-ring of subsets of a nonempty set $\Omega$. A subset $A$ of $c a(\Sigma)$ is relatively weakly compact if and only if $A$ is bounded and uniformly $\sigma$-additive.

Proof. By the Eberlein-Šmulian theorem and by the fact that for each sequence $\left(\mu_{n}\right) \subset c a(\Sigma)$ there exists $E \in \Sigma$ such that $\operatorname{var}\left(\mu_{n}, F\right)=0$ for each $F \in \Sigma$ with $F \cap E=\emptyset$ and for each $n$, we can replace the space $c a(S, \Sigma, \lambda)$ in the proof of Theorem IV.9.1 of [DS] by the space $c a(\Omega \cap E, \Sigma \cap E, \lambda)$ of all $\lambda$-continuous set functions in $c a(\Omega \cap E, \Sigma \cap E)$. Since $\Sigma \cap E$ is a $\sigma$-algebra, the rest of the argument in the proof of Theorem IV.9.1 of [DS] holds here to show that the conditions are necessary and sufficient.

\section{MAin Results}

In this section we obtain characterizations of (bounded) relatively weakly compact subsets of $M(T)$ in terms of the Baire and $\sigma$-Borel restrictions of the members of the set in question. These characterizations are similar to those obtained by Grothendieck in Theorem 2 of $[\mathrm{G}]$ and those of Lemma VI.2.13 of Diestel and Uhl [DU]. As mentioned in the introduction, these results are powerful enough to replace the use of Theorem 3 and Proposition 11 of $[\mathrm{G}]$ in our forthcoming paper [P3] where we characterize quasicomplete lcHs-valued weakly compact operators 
on $C_{o}(T)$. Moreover, the isolated results of Dinculeanu and Kluvánek [DK, K] on vector valued $\sigma$-additive Baire and Borel measures are deduced in [P3] as corollaries of some of these characterizations. Finally, Theorem 1 below combined with the study of Grothendieck on p.150 of [G] provides a generalization of Proposition 8 of Dieudonné [Die], which is stronger and more natural than that of Grothendieck [G]. See Corollary 1 below.

Theorem 1. Let $A$ be a bounded set in $M(T)$. Then the following statements are equivalent:

(i) A is relatively weakly compact.

(ii) For each disjoint sequence $\left(O_{i}\right)$ of open Baire sets in $T, \lim _{i} \mu\left(O_{i}\right)=0$ uniformly in $\mu \in A$.

(iii) For each disjoint sequence $\left(O_{i}\right)$ of open Baire sets in $T, \lim _{i}|\mu|\left(O_{i}\right)=0$ uniformly in $\mu \in A$.

(iv) a) $A$ is uniformly Baire inner regular in each open Baire set $O$ in $T$.

b) For each $\varepsilon>0$, there exists a $K \in \mathcal{C}_{0}(T)$ such that

$$
\sup _{\mu \in A}|\mu|(T \backslash K)<\varepsilon .
$$

(v) $A$ is uniformly Baire inner regular.

(vi) $\left.A\right|_{\mathcal{B}_{o}(T)}$ is uniformly $\sigma$-additive on $\mathcal{B}_{o}(T)$.

(vii) $A$ is uniformly Baire regular.

Proof. By Theorem 2 of Grothendieck [G] (which is the same as Theorem 4.22 .1 of Edwards [E]), (i) implies (ii).

(ii) $\Rightarrow$ (iii). Since each $\left.\mu\right|_{\mathcal{B}_{o}(T)}$ is Baire regular for $\mu \in A$, the argument in the proof of $(\mathrm{a}) \Rightarrow$ (b) of Lemma VI.2.13 of Diestel and Uhl [DU] can suitably be modified to show that (ii) $\Rightarrow$ (iii).

(iii) $\Rightarrow$ (iv). Let $O$ be an open Baire set in $T$ or let $O=T$. Let $\varepsilon>0$. If there exists no compact $G_{\delta} K \subset O$ such that $\sup _{\mu \in A}|\mu|(O \backslash K) \leq \varepsilon$, then there is a $\mu_{1} \in A$ such that $\left|\mu_{1}\right|(O)>\varepsilon$, for otherwise $K=\emptyset$ will provide a contradiction. If $O \in \mathcal{B}_{o}(T)$, then by the Baire regularity of $\mid \mu_{1} \|_{\mathcal{B}_{o}(T)}$ there exists a compact $G_{\delta}$ $K_{1} \subset O$ such that $\left|\mu_{1}\right|\left(K_{1}\right)>\varepsilon$. If $O=T$, then by the Borel regularity of $\left|\mu_{1}\right|$ there exists a compact $K$ such that $\left|\mu_{1}\right|(K)>\varepsilon$. Then by Theorem 50.D of Halmos [H] there exists a compact $G_{\delta} K_{1}$ such that $K \subset K_{1}$ and hence $\left|\mu_{1}\right|\left(K_{1}\right)>\varepsilon$. Since $K_{1}$ is a subset of $O$, again by Theorem 50.D of [H] there exist an open Baire set $O_{1}$ and a compact $G_{\delta} F_{1}$ such that

$$
O \supset F_{1} \supset O_{1} \supset K_{1} .
$$

Moreover, $\left|\mu_{1}\right|\left(O_{1}\right) \geq\left|\mu_{1}\right|\left(K_{1}\right)>\varepsilon$. Since $F_{1}$ is a compact $G_{\delta}$ subset of $O$, by our assumption there exists $\mu_{2} \in A$ such that $\left|\mu_{2}\right|\left(O \backslash F_{1}\right)>\varepsilon$. If $O \neq T$, then using the Baire regularity of $\left|\mu_{2}\right|$ in $O \backslash F_{1}$, and if $O=T$, then using the Borel regularity of $\left|\mu_{2}\right|$ in $O \backslash F_{1}$ and then applying Theorem 50.D of [H], we can choose a compact $G_{\delta} C_{1} \subset O \backslash F_{1}$ such that $\left|\mu_{2}\right|\left(C_{1}\right)>\varepsilon$. Let $K_{2}=F_{1} \cup C_{1}$. Then $K_{2}$ is a compact $G_{\delta}, O \supset K_{2} \supset F_{1}$ and $\left|\mu_{2}\right|\left(K_{2} \backslash F_{1}\right)=\left|\mu_{2}\right|\left(C_{1}\right)>\varepsilon$. Again by Theorem 50.D of [H] there exist an open Baire set $O_{2}$ and a compact $G_{\delta} F_{2}$ such that

$$
O \supset F_{2} \supset O_{2} \supset K_{2} \supset F_{1} \supset O_{1} \supset K_{1} \text {. }
$$

Accordingly, $\left|\mu_{2}\right|\left(O_{2} \backslash F_{1}\right) \geq\left|\mu_{2}\right|\left(K_{2} \backslash F_{1}\right)>\varepsilon$. Next, by our assumption there exists $\mu_{3} \in A$ such that $\left|\mu_{3}\right|\left(O \backslash F_{2}\right)>\varepsilon$. If $O \neq T$, then using the Baire regularity of $\left|\mu_{3}\right|$ 
in $O \backslash F_{2}$, and if $O=T$, then using the Borel regularity of $\left|\mu_{3}\right|$ in $O \backslash F_{2}$ and then applying Theorem 50.D of [H], we can choose a compact $G_{\delta} C_{2} \subset O \backslash F_{2}$ such that $\left|\mu_{3}\right|\left(C_{2}\right)>\varepsilon$. Let $K_{3}=F_{2} \cup C_{2}$. Then $K_{3}$ is a compact $G_{\delta}, O \supset K_{3} \supset F_{2}$ and $\left|\mu_{3}\right|\left(K_{3} \backslash F_{2}\right)=\left|\mu_{3}\right|\left(C_{2}\right)>\varepsilon$. Again by Theorem 50.D of $[\mathrm{H}]$ there exist an open Baire set $O_{3}$ and a compact $G_{\delta} F_{3}$ such that

$$
O \supset F_{3} \supset O_{3} \supset K_{3} \supset F_{2} \supset O_{2}
$$

and hence $\left|\mu_{3}\right|\left(O_{3} \backslash F_{2}\right) \geq\left|\mu_{3}\right|\left(K_{3} \backslash F_{2}\right)>\varepsilon$.

Proceeding as in the proof of (b) $\Rightarrow$ (c) of Lemma VI.2.13 of [DU], applying Theorem 50.D of $[\mathrm{H}]$ in each step and using the Baire regularity of each $\mid \mu \|_{\mathcal{B}_{o}(T)}$ for $\mu \in A$ or using the Borel regularuty of $\mu \in A$ and then applying Theorem 50.D of $[\mathrm{H}]$, as the case may be, we can produce an increasing sequence $\left(O_{n}\right)$ of open Baire sets in $T$, another two increasing sequences $\left(K_{n}\right)$ and $\left(F_{n}\right)$ of compact $G_{\delta} \mathrm{s}$ in $T$ and a sequence $\left(\mu_{n}\right)$ in $A$ such that

$$
O \supset \cdots \supset F_{n+1} \supset O_{n+1} \supset K_{n+1} \supset F_{n} \supset O_{n} \supset \cdots \supset K_{2} \supset F_{1} \supset O_{1} \supset K_{1}
$$

and

$$
\left|\mu_{n+1}\right|\left(O_{n+1} \backslash F_{n}\right)>\varepsilon
$$

for all $n \geq 1$. Let $G_{n+1}=O_{n+1} \backslash F_{n}, n \geq 1$. Then $\left(G_{n+1}\right)$ is a disjoint sequence of open Baire sets in $T$ and satisfies $\left|\mu_{n+1}\right|\left(G_{n+1}\right)>\varepsilon$ for $n \geq 1$. This contradicts (iii), and hence (iv) holds.

(iv) $\Rightarrow(\mathrm{v})$. Let $\varepsilon>0$. By (iv)(b) there exists a compact $G_{\delta} \Omega$ in $T$ such that

$$
\sup _{\mu \in A}|\mu|(T \backslash \Omega)<\frac{\varepsilon}{2} .
$$

We shall now modify the proof of (c) $\Rightarrow$ (d) of Lemma VI.2.13 of [DU] to show that (v) holds. Let $\mathcal{C}_{o}(\Omega)=\left\{K \subset \Omega: K\right.$ compact $G_{\delta}$ in $\Omega$ with respect to the relative topology of $\Omega$. It is easy to check that

$$
\mathcal{C}_{o}(\Omega)=\mathcal{C}_{o}(T) \cap \Omega=\left\{K \subset \Omega: K \in \mathcal{C}_{o}(T)\right\} .
$$

Let

$$
\begin{aligned}
\mathcal{S}= & \left\{E \in \mathcal{B}_{o}(\Omega): \text { for each } \varepsilon^{\prime}>0, \text { there exists } K \in \mathcal{C}_{o}(\Omega)\right. \\
& \text { such that } \left.E \cap K \text { is compact and } \sup _{\mu \in A}|\mu|(\Omega \backslash K) \leq \varepsilon^{\prime}\right\} .
\end{aligned}
$$

If $E \in \mathcal{B}_{o}(\Omega)$ and $K \in \mathcal{C}_{o}(\Omega)$ are such that $E \cap K$ is compact, then by Theorem 51.D of Halmos $[\mathrm{H}], E \cap K \in \mathcal{C}_{o}(\Omega)$. Clearly, $\mathcal{C}_{o}(\Omega) \subset \mathcal{S}$, since for $C \in \mathcal{C}_{o}(\Omega)$ we know that $C \cap \Omega=C$ is compact and $|\mu|(\Omega \backslash \Omega)=0$ for $\mu \in A$.

Claim 1. For each open Baire set $O$ in $T, O \cap \Omega \in \mathcal{S}$.

In fact, $O \cap \Omega \in \mathcal{B}_{o}(T) \cap \Omega=\mathcal{S}\left(\mathcal{C}_{o}(T)\right) \cap \Omega=\mathcal{S}\left(\mathcal{C}_{o}(T) \cap \Omega\right)=\mathcal{S}\left(\mathcal{C}_{o}(\Omega)\right)=\mathcal{B}_{o}(\Omega)$ by (3.2) and by Theorem 5.E of Halmos [H], where $\mathcal{S}(\mathcal{E})$ denotes the $\sigma$-ring generated by the class $\mathcal{E}$. Given $\varepsilon^{\prime}>0$, by (iv)(a) there exists $K \in \mathcal{C}_{o}(T)$ with $K \subset O$ such that

$$
\sup _{\mu \in A}|\mu|(O \backslash K) \leq \varepsilon^{\prime}
$$


Let $K_{o}=K \cap \Omega$. Then $K_{o} \in \mathcal{C}_{o}(\Omega)$ by (3.2), and moreover, $O \cap \Omega \cap K_{o}=K_{o}$ is compact. Further, as $(O \cap \Omega) \backslash K_{o} \subset O \backslash K$, by (3.3) we have

$$
\sup _{\mu \in A}|\mu|\left((O \cap \Omega) \backslash K_{o}\right) \leq \varepsilon^{\prime} .
$$

Let $K_{1}=K_{o} \cup(\Omega \backslash O)$. Then by Theorem 51.D of Halmos [H] and by (3.2), $\Omega \backslash O \in$ $\mathcal{C}_{o}(\Omega)$ and hence $K_{1} \in \mathcal{C}_{o}(\Omega)$. Moreover, $O \cap \Omega \cap K_{1}=K_{o}$ is compact, and by (3.4)

$$
\sup _{\mu \in A}|\mu|\left(\Omega \backslash K_{1}\right)=\sup _{\mu \in A}|\mu|\left((O \cap \Omega) \backslash K_{o}\right) \leq \varepsilon^{\prime} .
$$

Thus $O \cap \Omega \in \mathcal{S}$.

Claim 2. For $K \in \mathcal{C}_{o}(\Omega), \Omega \backslash K \in \mathcal{S}$.

In fact, by (3.2) $K$ is of the form $K=\bigcap_{1}^{\infty} V_{n}$, where the $V_{n}$ are open Baire sets in $T$ (see Proposition 14, $\S 14$, Chapter III of [Din]). Then $\Omega \backslash K=\bigcup_{1}^{\infty}\left(\Omega \backslash V_{n}\right)$. Now, by Theorem 50.D of Halmos [H] there exists an open Baire set $W_{n}$ in $T$ such that $\Omega \backslash V_{n} \subset W_{n}$ for $n \geq 1$. Let $W=\bigcup_{1}^{\infty} W_{n}$. Then $W$ is an open Baire set in $T$ and $\Omega \backslash K=(\Omega \backslash K) \cap W=\Omega \cap(W \backslash K)$. Since $W \backslash K$ is an open Baire set in $T$, by Claim 1 we conclude that $\Omega \backslash K \in \mathcal{S}$.

To show that $\mathcal{S}$ is closed under countable intersections, let $\left(E_{n}\right)$ be a sequence in $\mathcal{S}$ and let $\varepsilon^{\prime}>0$. Then, proceeding as on p.158 of [DU], we get a sequence $\left(K_{n}\right)$ in $\mathcal{C}_{o}(\Omega)$ such that $E_{n} \cap K_{n}$ is compact and $\sup _{\mu \in A}|\mu|\left(\Omega \backslash K_{n}\right) \leq \frac{\varepsilon^{\prime}}{2^{n}}$ for each $n \geq 1$. Then the set

$$
\left(\bigcap_{n=1}^{\infty} E_{n}\right) \cap\left(\bigcap_{n=1}^{\infty} K_{n}\right)=\bigcap_{n=1}^{\infty}\left(E_{n} \cap K_{n}\right)
$$

is compact and

$$
\sup _{\mu \in A}|\mu|\left(\Omega \backslash \bigcap_{1}^{\infty} K_{n}\right) \leq \sum_{n=1}^{\infty} \sup _{\mu \in A}|\mu|\left(\Omega \backslash K_{n}\right) \leq \varepsilon^{\prime} .
$$

Thus $\bigcap_{1}^{\infty} E_{n} \in \mathcal{S}$.

To verify that $\mathcal{S}$ is also closed under complements in $\Omega$, let $E \in \mathcal{S}$ and let $\varepsilon^{\prime}>0$. Then there exists $K_{1} \in \mathcal{C}_{o}(\Omega)$ such that $E \cap K_{1}$ is compact and $\sup _{\mu \in A}|\mu|\left(\Omega \backslash K_{1}\right) \leq$ $\frac{\varepsilon^{\prime}}{2}$. Now, by Claim 2 and by Theorem 51.D of [H], we have $\Omega \backslash\left(E \cap K_{1}\right) \in \mathcal{S}$. Therefore there exists $K_{2} \in \mathcal{C}_{o}(\Omega)$ such that $\left(\Omega \backslash\left(E \cap K_{1}\right)\right) \cap K_{2}$ is compact and $\sup _{\mu \in A}|\mu|\left(\Omega \backslash K_{2}\right) \leq \frac{\varepsilon^{\prime}}{2}$. Then $\left(K_{1} \cap K_{2}\right) \cap(\Omega \backslash E)=K_{1} \cap K_{2} \cap\left(\Omega \backslash\left(E \cap K_{1}\right)\right)$ is compact and

$$
\sup _{\mu \in A}|\mu|\left(\Omega \backslash\left(K_{1} \cap K_{2}\right)\right) \leq \sup _{\mu \in A}|\mu|\left(\Omega \backslash K_{1}\right)+\sup _{\mu \in A}|\mu|\left(\Omega \backslash K_{2}\right)<\varepsilon^{\prime} .
$$

Thus $\Omega \backslash E \in \mathcal{S}$. Consequently, $\mathcal{S}$ is a $\sigma$-algebra in $\Omega$.

Since $\mathcal{C}_{o}(\Omega) \subset \mathcal{S} \subset \mathcal{B}_{o}(\Omega)$, it follows that $\mathcal{S}=\mathcal{B}_{o}(\Omega)$. Thus, for each $E \in$ $\mathcal{B}_{o}(\Omega)$ and $\varepsilon^{\prime}>0$, there exists $K \in \mathcal{C}_{o}(\Omega)$ such that $E \cap K$ is compact and $\sup _{\mu \in A}|\mu|(\Omega \backslash K) \leq \varepsilon^{\prime}$. Then

$$
\sup _{\mu \in A}|\mu|(E \backslash(E \cap K)) \leq \sup _{\mu \in A}|\mu|(\Omega \backslash K) \leq \varepsilon^{\prime} .
$$

Now let $E \in \mathcal{B}_{o}(T)$ and $\varepsilon^{\prime}=\frac{\varepsilon}{2}$. Then $E \cap \Omega \in \mathcal{B}_{o}(\Omega)$ by (3.2) and by Theorem 5 .E of $[\mathrm{H}]$. Consequently, using $E \cap \Omega \in \mathcal{B}_{o}(\Omega)$ in place of $E$ above, as in (3.5) there 
exists $K \in \mathcal{C}_{o}(\Omega)$ such that $(E \cap \Omega) \cap K=K_{o}$ (say) is compact and

$$
\sup _{\mu \in A}|\mu|\left((E \cap \Omega) \backslash K_{o}\right) \leq \frac{\varepsilon}{2} .
$$

Thus $K_{o} \in \mathcal{C}_{o}(T), K_{o} \subset E$ and

$$
\sup _{\mu \in A}|\mu|\left(E \backslash K_{o}\right) \leq \sup _{\mu \in A}|\mu|\left((E \cap \Omega) \backslash K_{o}\right)+\sup _{\mu \in A}|\mu|(T \backslash \Omega)<\varepsilon
$$

by (3.6) and (3.1). Thus (v) holds.

Replacing in the proof of $(\mathrm{d}) \Rightarrow(\mathrm{e}) \Rightarrow$ (f) $\Rightarrow$ (a) of Lemma VI.2.13 of [DU] compact sets, Borel sets and open sets in $\Omega$ respectively by compact $G_{\delta}$ sets in $T$, Baire sets in $T$ and open Baire sets in $T$, one can easily show that $(\mathrm{v}) \Rightarrow(\mathrm{vi}) \Rightarrow$ (vii) $\Rightarrow$ (ii).

Finally, to show that (vi) $\Rightarrow$ (i), let $\Phi(\mu)=\left.\mu\right|_{\mathcal{B}_{o}(T)}$ for $\mu \in M(T)$ and let $\mathcal{M}_{o}(T)=\left\{\mu: \mathcal{B}_{o}(T) \rightarrow \mathbb{C}, \mu \sigma\right.$-additive $\}$ with $\|\mu\|_{o}=\sup _{E \in \mathcal{B}_{o}(T)}|\mu|(E)$ for $\mu \in \mathcal{M}_{o}(T)$. Then by Lemma 1 and by Theorem 5.3 of [P2], $\Phi$ is an isometric isomorphism of $M(T)$ onto $\mathcal{M}_{o}(T)$. By Proposition 1, and by (vi), $\Phi(A)$ is relatively weakly compact in $\mathcal{M}_{o}(T)$. Consequently, $A$ is relatively weakly compact in $M(T)$.

Corollary 1 (Generalization of Proposition 8 of Dieudonné [Die]). A bounded sequence $\left(\mu_{i}\right)$ in $M(T)$ is weakly convergent if and only if, for each open Baire set $O$ in $T, \lim _{i} \mu_{i}(O)$ exists in $\mathbb{C}$.

Proof. We only have to show that the condition is sufficient. By regularity, each complex Baire measure $\mu$ in $T$ is determined by its restriction on the lattice of all open Baire sets. Moreover, each complex Baire measure has a unique regular Borel extension. These facts and the Eberlein-Šmulian theorem ensure that it suffices to show that $\left(\mu_{i}\right)$ is relatively weakly compact in $M(T)$. Arguing as in the proof of Corollary 4.22.2 of Edwards [E], one can show that $\lim _{i} \mu_{n}\left(O_{i}\right)=0$ uniformly in $n \in \mathbb{N}$ for each disjoint sequence $\left(O_{i}\right)$ of open Baire sets in $T$. Then by the equivalence of (i) and (ii) of Theorem 1 it follows that $\left(\mu_{i}\right)$ is relatively weakly compact in $M(T)$.

Remark 1. When $T$ is compact, the proof of Proposition 9 in [Die] holds verbatim to show that the hypothesis that $\lim _{i} \mu_{i}(U)$ exists in $\mathbb{C}$ for each open set $U$ in $T$ implies that $\left(\mu_{i}\right)$ is bounded. When $T$ is locally compact, one can argue with its onepoint compactification as on p.177 of Thomas [T] to show that the above hypothesis also ensures the boundedness of $\left(\mu_{i}\right)$ in $M(T)$. Again, when $T$ is compact, using Theorem 50.D of $[\mathrm{H}]$ and the Baire regularity of the $\left.\mu_{i}\right|_{\mathcal{B}_{o}(T)}$ we can modify the proof of Proposition 9 in [Die] to show that $\left(\mu_{i}\right)$ is bounded when $\lim _{i} \mu_{i}(O)$ exists in $\mathbb{C}$ for each open Baire set $O$ in $T$. However, when $T$ is locally compact and not compact, we do not know whether the boundedness condition can be dispensed with in the above corollary. When $T$ is metrizable and compact $\mathcal{B}(T)=\mathcal{B}_{o}(T)$, and hence the above corollary reduces to Proposition 8 of Dieudonné [Die]. Thus the present generalization is more natural, and also stronger, than that of Grothendieck on p.150 of $[\mathrm{G}]$.

Theorem 2. Let $A$ be a bounded set in $M(T)$. Then the following statements are equivalent:

(i) A is relatively weakly compact. 
(ii) For each disjoint sequence $\left(O_{i}\right)$ of $\sigma$-Borel open sets (resp. (ii)'open sets) in $T$,

$$
\lim _{i} \mu\left(O_{i}\right)=0
$$

uniformly in $\mu \in A$.

(iii) For each disjoint sequence $\left(O_{i}\right)$ of $\sigma$-Borel open sets (resp. (iii)'open sets) in $T$,

$$
\lim _{i}|\mu|\left(O_{i}\right)=0
$$

uniformly in $\mu \in A$.

(iv) (a) $A$ is uniformly $\sigma$-Borel inner regular in each $\sigma$-Borel open set $O$ in $T$.

(b) For each $\varepsilon>0$, there exists a compact $K$ in $T$ such that

$$
\sup _{\mu \in A}|\mu|(T \backslash K) \leq \varepsilon .
$$

(resp. (iv) $)^{\prime} A$ is uniformly Borel inner regular in each open set $O$ in $T$ ).

(v) $A$ (resp. $\left.(v)^{\prime} A\right)$ is uniformly $\sigma$-Borel (resp. Borel) inner regular.

(vi) $\left.A\right|_{\mathcal{B}_{c}(T)}$ (resp. (vi)' $A$ ) is uniformly $\sigma$-additive on $\mathcal{B}_{c}(T)$ (resp. on $\mathcal{B}(T)$ ).

(vii) $A$ (resp. (vii)' $A$ ) is uniformly $\sigma$-Borel (resp. Borel) regular.

Proof. Let

$$
\mathcal{M}_{c}(T)=\left\{\mu: \mathcal{B}_{c}(T) \rightarrow \mathbb{C}, \mu \sigma \text {-additive and } \sigma \text {-Borel regular }\right\}
$$

with $\|\mu\|_{c}=\sup _{E \in \mathcal{B}_{c}(T)}|\mu|(E)$, and let $\Psi: M(T) \rightarrow \mathcal{M}_{c}(T)$ be given by $\Psi(\mu)=$ $\left.\mu\right|_{\mathcal{B}_{c}(T)}$. Then by Lemma 1 and by Theorem 5.3 of [P2], $\Psi$ is an isometric isomorphism of $M(T)$ onto $\mathcal{M}_{c}(T)$. This fact and an argument similar to that in the proof of Theorem 1 can be used to show that (i) $\Rightarrow$ (ii) (resp. (ii) $\left.{ }^{\prime}\right) \Rightarrow$ (iii) (resp. $\left.(\mathrm{iii})^{\prime}\right) \Rightarrow(\mathrm{iv})\left(\operatorname{resp} .(\mathrm{iv})^{\prime}\right) ;(\mathrm{v})\left(\operatorname{resp} .(\mathrm{v})^{\prime}\right) \Rightarrow(\mathrm{vi})\left(\operatorname{resp} .(\mathrm{vi})^{\prime}\right) \Rightarrow($ vii $\left.)(\text { resp. (vii) })^{\prime}\right)$ $\Rightarrow$ (ii) (resp. (ii) ${ }^{\prime}$ ) and (vi) (resp.(vi) $\left.)^{\prime}\right) \Rightarrow$ (i).

Now we shall prove (iv) (resp. (iv) $\left.)^{\prime}\right) \Rightarrow(\mathrm{v})$ (resp. (v)'). Given $\varepsilon>0$, by (iv)(b) (resp. by (iv) ${ }^{\prime}$ ) there exists a compact set $\Omega$ in $T$ such that

$$
\sup _{\mu \in A}|\mu|(T \backslash \Omega)<\frac{\varepsilon}{2} .
$$

Let

$$
\begin{aligned}
\Sigma= & \left\{E \in \mathcal{B}(\Omega): \text { for each } \varepsilon^{\prime}>0, \text { there exists a compact } K \subset \Omega\right. \\
& \text { such that } \left.E \cap K \text { is compact and } \sup _{\mu \in A}|\mu|(\Omega \backslash K) \leq \varepsilon^{\prime}\right\} .
\end{aligned}
$$

Clearly, $\mathcal{C}(\Omega)=\{K \subset \Omega: K \quad$ compact $\} \subset \Sigma$.

We claim that $O \cap \Omega \in \Sigma$ for each $\sigma$-Borel open set (resp. open set) $O$ in $T$. In fact, given $\varepsilon^{\prime}>0$, by (iv)(a) (resp. (iv) $)^{\prime}$ there exists a compact $K$ in $T$ with $K \subset O$ such that $\sup _{\mu \in A}|\mu|(O \backslash K) \leq \varepsilon^{\prime}$. Let $K_{o}=K \cap \Omega$. Then $O \cap \Omega \cap K_{o}=K_{o}$ is compact, and clearly $O \cap \Omega \in \mathcal{B}(\Omega)$. Moreover,

$$
\sup _{\mu \in A}|\mu|\left((O \cap \Omega) \backslash K_{o}\right) \leq \sup _{\mu \in A}|\mu|(O \backslash K) \leq \varepsilon^{\prime} .
$$

Setting $K_{1}=K_{o} \cup(\Omega \backslash O)$, we note that $K_{1}$ is compact , $K_{1} \subset \Omega,(\Omega \cap O) \cap K_{1}=$ $K_{o} \in \mathcal{C}(T)$ and $\sup _{\mu \in A}|\mu|\left(\Omega \backslash K_{1}\right) \leq \varepsilon^{\prime}$. Thus $O \cap \Omega \in \Sigma$.

We also claim that $\Omega \backslash K \in \Sigma$ for each compact $K \subset \Omega$. In fact, by Theorem 50.D of $[\mathrm{H}]$ there exists a relatively compact open set $U$ in $T$ such that $\Omega \subset U$. Clearly 
$U$ is a $\sigma$-Borel open set in $T$, and $\Omega \backslash K=(\Omega \backslash K) \cap U=\Omega \cap(U \backslash K)$ with $U \backslash K$ a $\sigma$-Borel open set in $T$. Then by the foregoing claim it follows that $\Omega \backslash K \in \Sigma$.

Proceeding as on p.158 of [DU], one can show that $\Sigma$ is closed under countable intersections. The argument used in the proof of (iv) $\Rightarrow(\mathrm{v})$ of Theorem 1 to show that $\mathcal{S}$ is closed under complements can be modified here to prove that $\Sigma$ is also closed under complements in $\Omega$. Thus $\Sigma$ is a $\sigma$-algebra in $\Omega$. As $\mathcal{C}(\Omega) \subset \Sigma \subset \mathcal{B}(\Omega)$, it follows that $\Sigma=\mathcal{B}(\Omega)$. Then arguing as in the last part of (iv) $\Rightarrow(\mathrm{v})$ of Theorem 1 but using (3.7) in place of (3.1), we conclude that (v) (resp. (v)') holds.

\section{REFERENCES}

[BDS] R.G. Bartle, N. Dunford, and J.T. Schwartz, Weak compactness and vector measures, Canad. J.Math. 7, (1955), 289-305. MR 16:1123c

[DU] J. Diestel, and J.J. Uhl, Vector Measures, Survey No.15, Amer. Math. Soc. Providence, R.I., (1977). MR 56:12216

[Die] J. Dieudonné, Sur la convergence des suites de mesures de Radon, Anais Acad. Bras. Ciencias 23, (1951), 21-38. MR 13:121a

[Din] N. Dinculeanu, Vector Measures, Pergamon Press, New York, (1967). MR 34:6011

[DK] N. Dinculeanu, and I. Kluvánek, On vector measures, Proc. London Math. Soc. 17, (1967), 505-512. MR 35:5571

[DS] N. Dunford, and J.T. Schwartz, Linear Operators, Part I, General Theory, Interscience, New York, (1958). MR 22:8302

[E] R. E. Edwards, Functional Analysis, Theory and Applications, Holt, Rinehart and Winston, New York, (1965). MR 36:4308

[G] A. Grothendieck, Sur les applications linéares faiblement compactes d'espaces du type $C(K)$, Canad. J. Math. 5, (1953), 129-173. MR 15:438b

[H] P. R. Halmos, Measure Theory, Van Nostrand, New York, (1950). MR 11:504d

[K] I. Kluvánek, Characterization of Fourier-Stieltjes transforms of vector and operator valued measures, Czech. Math. J. 17, (1967), 261-277. MR 37:6430

[P1] T.V. Panchapagesan, On complex Radon measures I, Czech. Math. J. 42, (1992), 599-612. MR 94c: 28014

[P2] _ On complex Radon measures II, Czech. Math. J. 43, (1993), 65-82. MR 94i:28011

[P3] Characterizations of weakly compact operators on $C_{o}(T)$, Trans. Amer. Math. Soc. 350 (1998), 4849-4867.

[T] E. Thomas, L'intégration par rapport a une mesure de Radon vectorielle, Ann. Inst. Fourier (Grenoble) 20, (1970), 55-191. MR 57:3348

Departamento de Matemáticas, Facultad de Ciencias, Universidad de los Andes, MÉRIDA, VENEZuEla

E-mail address: panchapa@ciens.ula.ve 